\title{
Supporting Information \\ Addressing the Origin of Photocurrents and Fuel Production Activities \\ in Catalyst-Modified Semiconductor Electrodes
}

Brian L. Wadsworth, Nghi P. Nguyen, Daiki Nishiori, Anna M. Beiler, and Gary F. Moore*

School of Molecular Sciences and the Biodesign Institute Center for Applied Structural Discovery (CASD)

Arizona State University, Tempe, AZ 85287-1604, United States

gfmoore@asu.edu

Index

$\begin{array}{ll}\text { 1. Additional Experimental Methods } & \text { S2 }\end{array}$

2. Definitions of Key Terms $\quad$ S4

3. Reaction Schemes for Photoelectrosynthesis, Screening, and Dye-Sensitized Hole Injection S5

4. Optical Characterization Data $\quad$ S6

$\begin{array}{llr}\text { 5. } & \text { (Photo)electrochemical Data } & \text { S7 }\end{array}$

6. Absorbance Spectra and Wavelength-Resolved Light Harvesting Efficiency Plots S9

7. External Quantum Efficiency Action Spectra $\quad$ S12

8. Spectral Profile of the LSC-100 Series Oriel Solar Simulator $\quad$ S15

9. Supporting Information References $\quad$ S16 


\section{Additional Experimental Methods}

\section{Synthesis}

$\mathbf{5 , 1 0 , 1 5 , 2 0 - t e t r a - p - t o l y l p o r p h y r i n . ~ A ~ s i m i l a r ~ m e t h o d ~ w a s ~ p r e v i o u s l y ~ r e p o r t e d . ~}{ }^{1,2}$ A solution of 5-(4-methylphenyl)dipyrromethane $(1.417 \mathrm{~g}, 6 \mathrm{mmol})$ and $p$-tolyl aldehyde $(707 \mu \mathrm{L}, 6 \mathrm{mmol})$ in chloroform $(600 \mathrm{~mL})$ was purged for $15 \mathrm{~min}$ with argon before adding $\mathrm{BF}_{3} \cdot \mathrm{OEt}_{2}(792 \mu \mathrm{L}$ of $2.5 \mathrm{M}$ stock solution in chloroform, final concentration was $3.3 \mathrm{mM})$. After stirring for $12 \mathrm{~h}, 2,3$-dichloro-5,6-dicyano-1,4-benzoquinone $(2.04 \mathrm{~g}, 9 \mathrm{mmol})$ was added and the mixture was stirred for an additional $3 \mathrm{~h}$ before adding $7.5 \mathrm{~mL}$ of triethylamine. The mixture was filtered through a pad of alumina and the solvent was evaporated at reduced pressure. The crude product was purified via column chromatography on silica using a mixture of 2:1 chloroform and hexanes as the eluent to yield the desired product $(45 \%) .{ }^{1} \mathrm{H} \mathrm{NMR}\left(400 \mathrm{MHz}, \mathrm{CDCl}_{3}\right): \delta-2.77(2 \mathrm{H}, \mathrm{s}, \mathrm{NH})$, $2.70\left(12 \mathrm{H}, \mathrm{s}, \mathrm{CH}_{3}\right), 7.55(8 \mathrm{H}, \mathrm{d}, J=7.8 \mathrm{~Hz}, \mathrm{ArH}), 8.09(8 \mathrm{H}, \mathrm{d}, J=7.8 \mathrm{~Hz}, \mathrm{ArH}) 8.85(8 \mathrm{H}, \mathrm{s}, \beta \mathrm{H})$; MALDI-TOF-MS $m / z$ calcd. for $\mathrm{C}_{48} \mathrm{H}_{38} \mathrm{~N}_{4} 670.31$, obsd. 670.46; UV-vis-NIR (Toluene) 420, 516, 550, 593, $651 \mathrm{~nm}$.

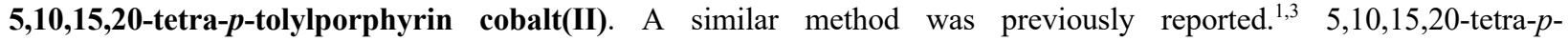
tolylporphyrin ( $25 \mathrm{mg}, 0.03 \mathrm{mmol}$ ) and cobalt (II) acetate (53 mg, $0.3 \mathrm{mmol}$ ) were dissolved in dimethylformamide (35 mL) and brought to reflux. The mixture was refluxed for $20 \mathrm{~min}$ before evaporating the solvent at reduced pressure. The product was purified on an alumina column using dichloromethane as the eluent and the product was recrystallized from dichloromethane/methanol to give the desired compound (98\%). UV-vis-NIR (Toluene) 416, $530 \mathrm{~nm}$.

\section{CoTTP $\mid$ PPy $\mid$ GaP Sample Preparation}

Zn-doped p-type gallium phosphide (GaP) (100) wafers were purchased from the Institute of Electronic Materials Technology (ITME). The single-side-polished, crystalline wafers had a $400 \mu \mathrm{m}$ thickness, a resistivity of $0.053-0.045 \Omega$ $\mathrm{cm}$, a carrier concentration of $1.8-2.3 \times 10^{18} \mathrm{~cm}^{-3}$, and an etch pit density of less than $2 \times 10^{4} \mathrm{~cm}^{-2}$.

Diced semiconductor samples were degreased by wiping the surface with an acetone-soaked cotton swab and then ultrasonically cleaned in acetone followed by clean-room isopropanol for $5 \mathrm{~min}$ each, before drying under nitrogen. These samples were then exposed to an air-generated oxygen plasma (Harrick Plasma, U.S.) at $30 \mathrm{~W}$ for 2 min and surface oxide layers were removed by exposing the plasma-treated samples to buffered hydrofluoric acid $\left(6: 1 \mathrm{HF} / \mathrm{NH}_{4} \mathrm{~F}\right.$ in $\left.\mathrm{H}_{2} \mathrm{O}\right)$ for $5 \mathrm{~min}$, followed by rinsing first with $18.2 \mathrm{M} \Omega \mathrm{cm}$ water and then distilled methanol before drying under a stream of nitrogen. Next, the freshly etched wafers were placed in a quartz reaction vesicle containing argon-sparged 4-vinylpyridine and exposed to $254 \mathrm{~nm}$ UV light for $2 \mathrm{~h}$. After rinsing with methanol, the wafers were dried under nitrogen and stored under vacuum. The polypyridyl-functionalized GaP samples were then soaked for $18 \mathrm{~h}$ in a $1 \mathrm{mM}$ solution of 5,10,15,20-tetra-p-tolylporphyrin cobalt (II) in toluene. The cobalt porphyrin-polypyridyl-functionalized GaP samples were then rinsed with toluene followed by clean-room isopropanol and dried under a stream of nitrogen.

$\mathrm{GaP}$ and chemically-modified $\mathrm{GaP}$ working electrodes were fabricated by applying an indium-gallium eutectic (Aldrich) to the unpolished/backside of the wafers, then fixing a copper wire to the backside of the wafer using a conductive silver epoxy (Circuit Works). The copper wire was passed through a glass tube, and the wafer was insulated and attached to the glass tube with Loctite 615 Hysol Epoxi-patch adhesive. The epoxy was allowed to cure for approximately $24 \mathrm{~h}$ before performing experiments using the electrodes.

The GaP ingot used to prepare the samples described in this report has different physical characteristics (doping density, charge mobility, and resistance (see Experimental Section in the main text for details) than those used to prepare samples described in previous reports from our group. ${ }^{1,-8}$ For the polymer-modified electrodes assembled from these wafers, the polypyridyl coatings are $4 \pm 1 \mathrm{~nm}$ thick as determined using spectroscopic ellipsometry. X-ray photoelectron spectroscopy and inductively coupled plasma mass spectrometry measurements indicate $25 \%$ of the pyridyl sites are coordinated to a cobalt center following the porphyrin attachment chemistry, ${ }^{4}$ yielding a per geometric area surface concentration of cobalt of approximately $1 \mathrm{nmol} \mathrm{cm}$.

UV-Vis-NIR

Spectra of 5,10,15,20-tetra-p-tolylporphyrin cobalt(II). Aliquots taken from a stock solution of 5,10,15,20-tetra- $p$ tolylporphyrin cobalt(II) (CoTTP) in chloroform $(5.5 \mu \mathrm{M})$ were diluted with chloroform to prepare solutions at five additional concentrations $(0.55 \mu \mathrm{M}, 1.1 \mu \mathrm{M}, 2.2 \mu \mathrm{M}, 3.3 \mu \mathrm{M}$ and $4.4 \mu \mathrm{M})$. An absorption spectrum of each solution was collected using a quartz cuvette (pathlength of $1 \mathrm{~cm}$ ) sealed under air, and associated extinction coefficients were obtained from the slope of an absorbance versus concentration plot. This entire process was carried out three times using three different stock solutions of CoTTP in chloroform $(5.5 \mu \mathrm{M})$, and the extinction coefficients were reported as averaged values.

Spectra of 5,10,15,20-tetra-p-tolylporphyrin cobalt(II) with 1 Equivalent of Pyridine. A mixture of 5,10,15,20-tetrap-tolylporphyrin cobalt(II) (CoTTP) in chloroform with $\sim 1$ equivalent of pyridine per porphyrin was prepared by mixing 2 $\mathrm{mL}$ of $9 \mu \mathrm{M}$ CoTTP in chloroform with $2 \mathrm{~mL}$ of $11 \mu \mathrm{M}$ pyridine in chloroform and adjusting the volume of the mixture to $5 \mathrm{~mL}$ in total by adding chloroform. Absorption spectra of this mixture were recorded at varying time intervals $(5 \mathrm{~min}, 1 \mathrm{~h}$, $2 \mathrm{~h}, 7 \mathrm{~h}, 10 \mathrm{~h}$, and $12 \mathrm{~h}$ after the addition of $\sim 1$ equivalent of pyridine) using a quartz cuvette (pathlength of $1 \mathrm{~cm}$ ) sealed 
under air. The extinction coefficients of the complex formed after 26 hours (assigned as a CoTTP-pyridine- $\mathrm{O}_{2}$ complex) co,10 $^{2}$ were obtained by multiplying the extinction coefficients determined at selected wavelengths in the absence of pyridine by the ratio of the absorbance values measured at the same selected wavelengths prior to and following $26 \mathrm{~h}$ after the addition of pyridine. This entire process was repeated two additional times, and the extinction coefficients were reported as averaged values.

Solid-State Optical Measurements. All transmittance and reflectance measurements were performed using an integrating sphere, and data were collected at $10 \mathrm{~nm}$ intervals. Specular reflectance measurements were collected at an incident angle of $5^{\circ}$ to the normal of the surface using a specular reflectance attachment accessory from Shimadzu and a STAN-SSHreference mirror from OceanOptics. Absorption coefficients for GaP and chemically-modified GaP samples were calculated using Equation S1:

$\alpha=-\ln \left[\frac{(\% T+\% R)}{100}\right] / L$

where $\alpha$ is the wavelength-dependent absorption coefficient, $L$ is the pathlength (which is the $400 \mu \mathrm{m}$ thickness of the GaP wafers), $\% T$ is the percent transmittance collected at normal incidence, and $\% R$ is the sum of the diffuse percent reflectance and specular percent reflectance measured at an incident angle of $5^{\circ}$ to the normal of the surface.

\section{Photoelectrochemical Measurements}

All electrochemical measurements were performed using a Biologic potentiostat equipped with a platinum coil counter electrode, a $\mathrm{Ag} / \mathrm{AgCl}, \mathrm{NaCl}(3 \mathrm{M})$ reference electrode $(0.21 \mathrm{~V}$ vs NHE), and $\mathrm{GaP}$ working electrodes in a modified cell containing a quartz window. Linear sweep voltammograms were recorded in the dark and under $100 \mathrm{~mW} \mathrm{~cm}^{-2}$ illumination using a $100 \mathrm{~W}$ Oriel Solar Simulator equipped with an air mass 1.5 filter. The supporting electrolyte was $0.1 \mathrm{M}$ phosphate buffer ( $\mathrm{pH} 7$ ). Linear sweep voltammograms were recorded at a scan rate of $100 \mathrm{mV} \mathrm{s}^{-1}$ under a continuous flow of $5 \%$ hydrogen in nitrogen.

\section{Quantum Efficiency Measurements}

External Quantum Efficiency. External quantum efficiencies were measured under a bias of $0 \mathrm{~V}$ vs RHE (where E vs RHE $=\mathrm{E}$ vs NHE $+0.05916 \mathrm{~V} \times \mathrm{pH}=\mathrm{E}$ vs Ag/AgCl $+0.05916 \mathrm{~V} \times \mathrm{pH}+0.21 \mathrm{~V}$ ) in the wavelength range of 300-750 nm. The experimental setup consisted of a Biologic potentiostat and a QEPVSI-b Quantum Efficiency Measurement System from Newport Corporation, including a reference detector with NIST-traceable responsivity data, a $300 \mathrm{~W}$ Oriel Xenon lamp, and an Oriel Cornerstone 260 monochromator. Data were collected at $10 \mathrm{~nm}$ intervals, with a $30 \mathrm{~s}$ pause between steps. The current was collected at one point per second, and the data collected at each wavelength were averaged. The dark current was subtracted from the current produced under illumination. The photon flux $\left(q_{\mathrm{p}}\right)$ was determined using the voltage response of the reference detector in accordance with Equation S2:

$q_{\mathrm{p}}=\frac{(\mathrm{V})}{(\text { CR })(\text { SR })(\text { Gain }) E(\lambda)}$

where $V$ is the detector voltage, $C R$ is the correction multiplier of the AC measurement, $S R$ is the spectral responsivity of the reference detector, Gain is the gain set for the detector, and $E$ is the energy of a photon at a given wavelength. For the normalized action spectra, the data were normalized to the maximum absorbance $(300 \mathrm{~nm})$, a wavelength outside of the cobalt porphyrin Soret and Q-type absorption maxima.

Internal Quantum Efficiency. Internal quantum efficiencies were calculated by dividing the external quantum efficiency at each wavelength by the light harvesting efficiency at each wavelength. The light harvesting efficiency at each wavelength was determined using UV-vis-NIR absorption spectroscopy and Equations S3 and S4:

$\Phi_{\mathrm{abs}}=\mathrm{LHE}=1-10^{-A}$

$A=\frac{\alpha * L}{\ln 10}$

where $A$ is the absorbance, $\alpha$ is the wavelength-dependent absorption coefficient, and $L$ is the pathlength or thickness of the $\mathrm{GaP}$ wafer $(400 \mu \mathrm{m})$. 


\section{Definitions of Key Terms}

External quantum efficiency (EQE): also known as incident photon-to-current efficiency (IPCE), is the ratio of electron flux measured as current to incident photon flux. EQE can also be defined as the product of the probability of absorption, $\left(\Phi_{\text {abs }}\right)$, probability of charge separation $\left(\Phi_{\mathrm{sep}}\right)$, and probability of charge injection $\left(\Phi_{\text {inj }}\right)$.

$\mathrm{EQE}=\mathrm{IPCE}=\Phi_{\mathrm{abs}} \Phi_{\mathrm{sep}} \Phi_{\text {inj }}=\frac{\text { electrons } \mathrm{cm}^{-2} \mathrm{~s}^{-1}}{\text { incident photons } \mathrm{cm}^{-2} \mathrm{~s}^{-1}}$

Light harvesting efficiency (LHE): is the fraction of incident photons absorbed by a material. LHE is synonymous with the terms absorptance and $\Phi_{\text {abs, }}$, thus LHE is a component of EQE.

$\mathrm{LHE}=\Phi_{\mathrm{abs}}=\frac{\text { absorbed photons } \mathrm{cm}^{-2} \mathrm{~s}^{-1}}{\text { incident photons } \mathrm{cm}^{-2} \mathrm{~s}^{-1}}$

Internal quantum efficiency (IQE): also known as absorbed photon-to-current efficiency (APCE), is the ratio of electron flux measured as current to absorbed photon flux. IQE can also be defined as the product of $\Phi_{\text {sep }}$ and $\Phi_{\text {inj }}$, which is equal to the EQE divided by $\Phi_{\text {abs. }}$.

$\mathrm{IQE}=\mathrm{APCE}=\frac{\mathrm{EQE}}{\Phi_{\mathrm{abs}}}=\Phi_{\mathrm{sep}} \Phi_{\mathrm{inj}}=\frac{\text { electrons } \mathrm{cm}^{-2} \mathrm{~s}^{-1}}{\text { absorbed photons } \mathrm{cm}^{-2} \mathrm{~s}^{-1}}$

Dye-contribution efficiency (DCE): is the probability that photons absorbed by dye molecules contribute to photocurrent generation. ${ }^{11}$

$\mathrm{DCE}=\frac{\text { electrons } \mathrm{cm}^{-2} \mathrm{~s}^{-1}}{\text { absorbed photons } \mathrm{cm}^{-2} \mathrm{~s}^{-1}}$

EQE, LHE, IQE, and DCE are defined at a specified wavelength. However, they can also be described as a single parameter integrated over a specified spectral range. 


\section{Optical Characterization Data}
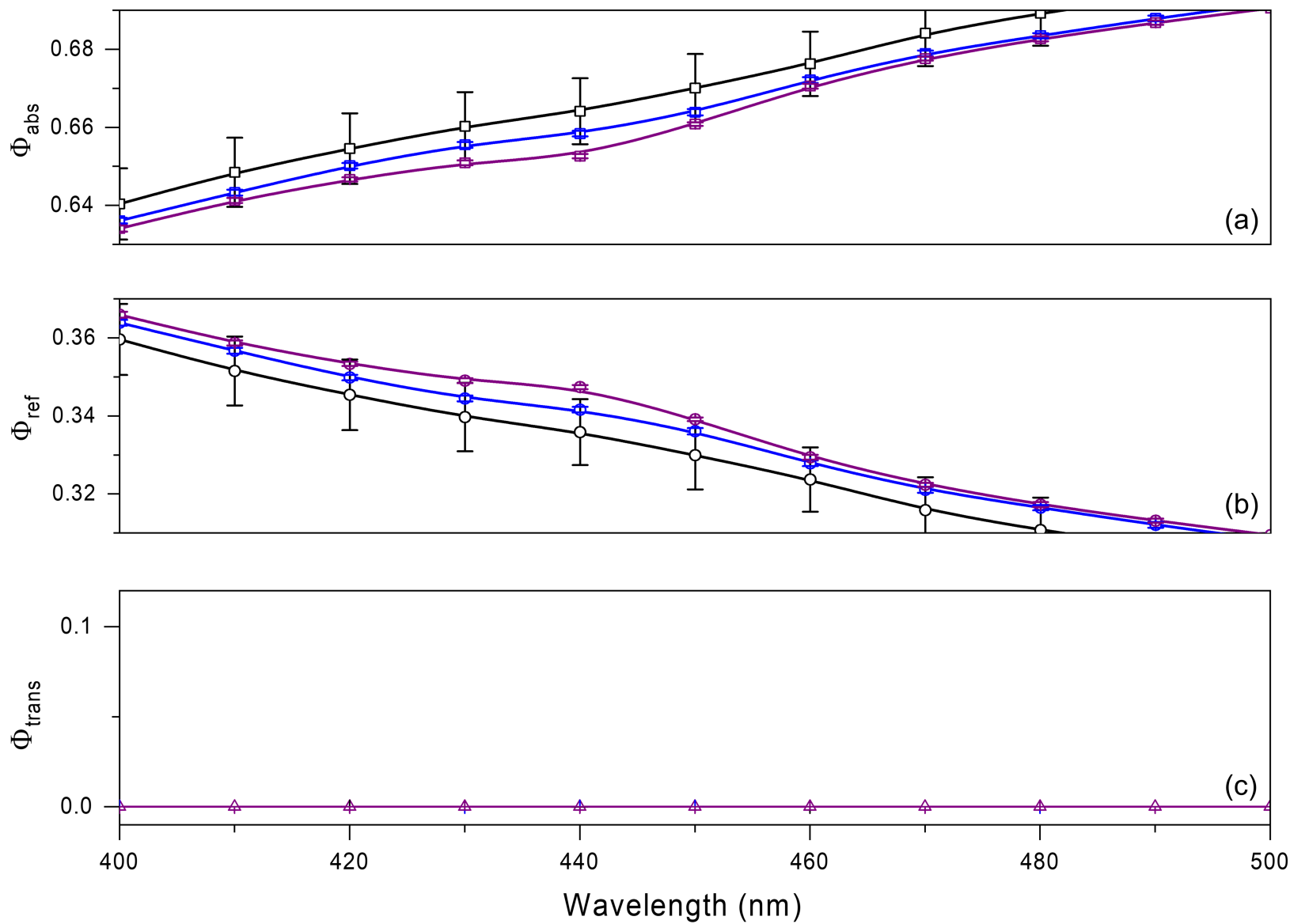

Figure S1. Plots of the (a) probability of absorption $\left(\Phi_{\text {abs }}\right)$ (squares), (b) probability of reflection ( $\left.\Phi_{\text {ref }}\right)$ (circles), and (c) probability of transmission ( $\Phi_{\text {trans }}$ ) (triangles) measured using GaP (black), PPy $\mid$ GaP (blue), and CoTTP $\mid$ PPy $\mid \mathrm{GaP}$ (purple) versus the wavelength of illumination. 


\section{5. (Photo)electrochemical Data}

Table S1. Open-circuit voltages, short-circuit current densities, maximum power points, and fill factors recorded using GaP, $\mathrm{PPy} \mid \mathrm{GaP}$, or CoTTP $|\mathrm{PPy}| \mathrm{GaP}$ electrodes in $0.1 \mathrm{M}$ phosphate buffer $(\mathrm{pH} 7)$ under broadband simulated solar illumination.

\begin{tabular}{c|c|c|c|c}
\hline Construct & $\begin{array}{c}\text { Open-Circuit Voltage } \\
\text { (V vs RHE) }\end{array}$ & $\begin{array}{c}\text { Short-Circuit Current } \\
\left(\mathbf{m} \mathbf{~ c m}^{-2}\right)\end{array}$ & $\begin{array}{c}\text { Maximum Power Point } \\
\left(\mathbf{m W} \mathbf{~ c m}^{-2}\right)\end{array}$ & Fill Factor \\
\hline $\mathbf{G a P}$ & $0.57 \pm 0.03$ & $0.50 \pm 0.07$ & $0.03 \pm 0.01$ & $0.10 \pm 0.03$ \\
$\mathbf{P P y} \mid \mathbf{G a P}$ & $0.66 \pm 0.01$ & $0.68 \pm 0.06$ & $0.06 \pm 0.02$ & $0.13 \pm 0.02$ \\
$\mathbf{C o T T P}|\mathbf{P P y}| \mathbf{G a P}$ & $0.67 \pm 0.01$ & $0.84 \pm 0.04$ & $0.14 \pm 0.04$ & $0.25 \pm 0.04$
\end{tabular}

Table S2. Current densities of PPy $\mid \mathrm{GaP}$ or CoTTP $|\mathrm{PPy}| \mathrm{GaP}$ electrodes polarized at $0 \mathrm{~V}$ vs RHE in $0.1 \mathrm{M}$ phosphate buffer ( $\mathrm{pH} 7$ ) determined using three different methods.

\begin{tabular}{|c|c|c|c|}
\hline Construct & $\begin{array}{c}\text { Current Density } \\
\text { at } 0 \text { V vs RHE }\left(\mathrm{mA} \mathrm{cm}^{-2}\right) \\
\begin{array}{c}\text { Broadband: Solar Simulator } \\
\text { with AM 1.5 Filter }\end{array} \\
\end{array}$ & $\begin{array}{c}\text { Current Density } \\
\text { at } 0 \text { V vs RHE }\left(\mathrm{mA} \mathrm{cm}^{-2}\right) \\
\text { Wavelength-Resolved: } \\
\text { AM 1.5 G Spectrum } \\
\end{array}$ & $\begin{array}{c}\text { Current Density } \\
\text { at } 0 \text { V vs RHE }\left(\mathrm{mA} \mathrm{cm}^{-2}\right) \\
\text { Wavelength-Resolved: Solar } \\
\text { Simulator with AM 1.5 Filter } \\
\end{array}$ \\
\hline $\mathbf{P P y} \mid \mathrm{GaP}$ & $0.68 \pm 0.06$ & $0.71 \pm 0.06$ & $0.77 \pm 0.06$ \\
\hline CoTTP|PPy|GaP & $0.84 \pm 0.04$ & $0.83 \pm 0.05$ & $0.90 \pm 0.05$ \\
\hline
\end{tabular}

${ }^{a}$ Current densities recorded via linear sweep voltammetry experiments performed under broadband simulated solar illumination

${ }^{b}$ Current densities calculated via integration of electron flux action spectra that were constructed using EQE action spectra and the air mass 1.5 global tilt (AM $1.5 \mathrm{G})$ spectrum $^{12}$

${ }^{c}$ Current densities calculated via integration of electron flux action spectra that were constructed using EQE action spectra and the photon flux associated with the spectral profile of the solar simulator 

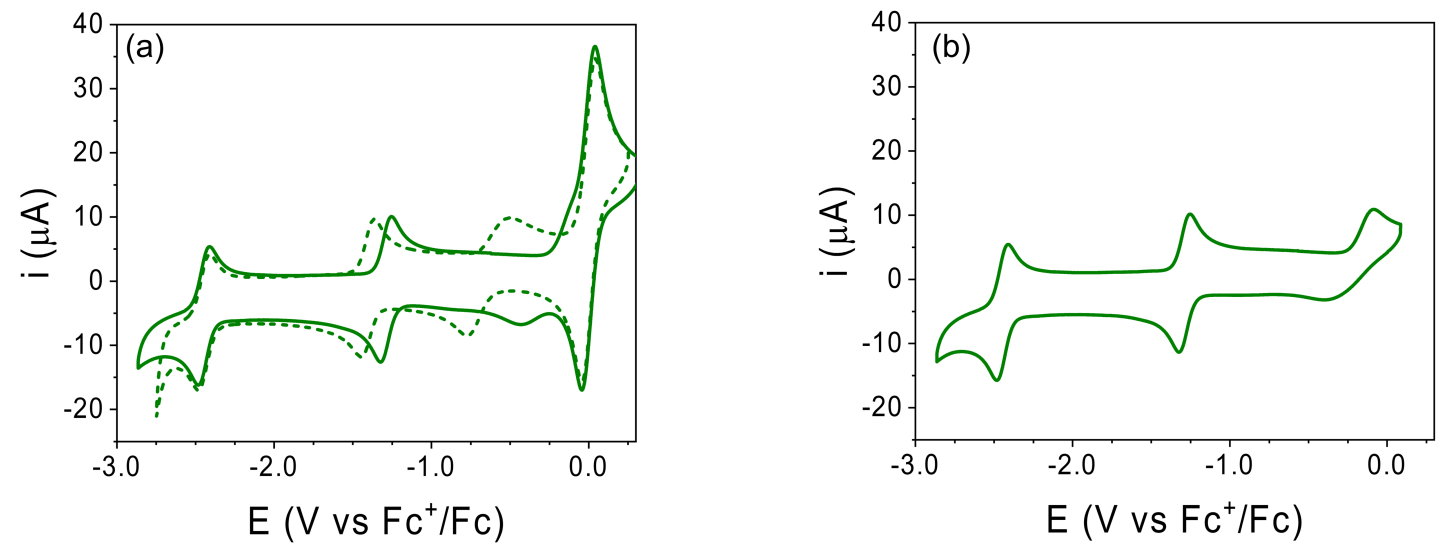

Figure S2. (a) Cyclic voltammograms of 5,10,15,20-tetra- $p$-tolylporphyrin cobalt(II) (CoTTP) (0.34 mM) recorded in dimethylformamide containing $0.1 \mathrm{M}$ tetrabutylammonium hexafluorophosphate $\left(\mathrm{TBAPF}_{6}\right)$ as the supporting electrolyte, ferrocene as an internal standard, and 0 (solid green) or 2,282 (dashed green) equivalents of pyridine. (b) Cyclic voltammogram of CoTTP recorded in dimethylformamide containing $0.1 \mathrm{M} \mathrm{TBAPF}_{6}$ in the absence of ferrocene. This voltammogram is included for comparison given the overlap between the ferrocenium/ferrocene $\left(\mathrm{Fc}^{+} / \mathrm{Fc}\right)$ redox couple and the redox feature assigned to the Co ${ }^{\mathrm{III} / \mathrm{II}}$ couple of CoTTP. All voltammograms were collected at room temperature under an argon atmosphere using a $3 \mathrm{~mm}$ diameter glassy carbon working electrode and referenced to the ferrocenium/ferrocene redox couple.

Table S3. Redox potentials associated with the reduction $\left({ }^{\mathrm{nI}} E\right)$ and oxidation $\left({ }^{\mathrm{ni}} E\right)$ of $5,10,15,20$-tetra- $p$-tolylporphyrin cobalt(II) (CoTTP) in the absence or presence of pyridine as determined by cyclic voltammetry. Peak-to-peak separations $\left(\Delta E_{\mathrm{p}}\right)$ are reported for electrochemically reversible, or quasi-reversible, redox processes in parentheses. For electrochemically irreversible redox couples, the anodic peak potential and cathodic peak potential are reported. All voltammograms were recorded in dimethylformamide containing $0.1 \mathrm{M} \mathrm{TBAPF}_{6}$ as the supporting electrolyte under argon using a $3 \mathrm{~mm}$ diameter glassy carbon working electrode at room temperature and the ferrocenium/ferrocene redox couple as an internal reference.

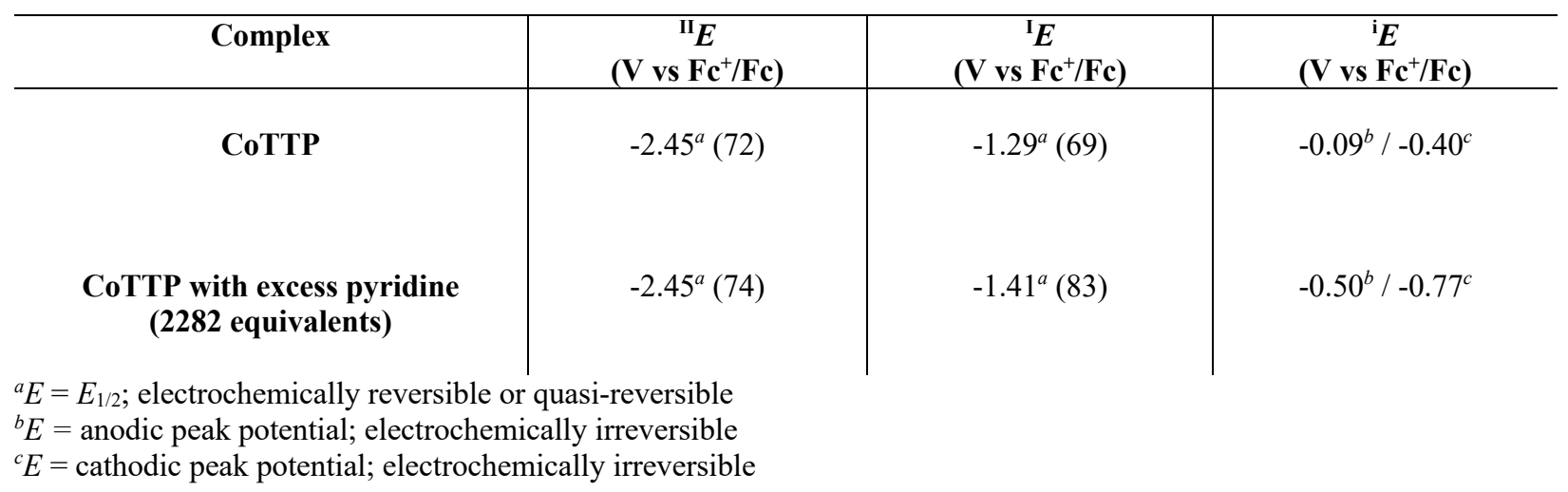




\section{Absorbance Spectra and Wavelength-Resolved Light Harvesting Efficiency Plots}

Scheme S2. Schematic representation of equilibria involving CoTTP, CoTTP-pyridine, and CoTTP-pyridine-O ${ }_{2}$ In this scheme, oxygen binds to the cobalt center of CoTTP-pyridine to form a CoTTP-pyridine- $\mathrm{O}_{2}$ complex, where the oxygen exists formally as a superoxide ion. ${ }^{9,10}$
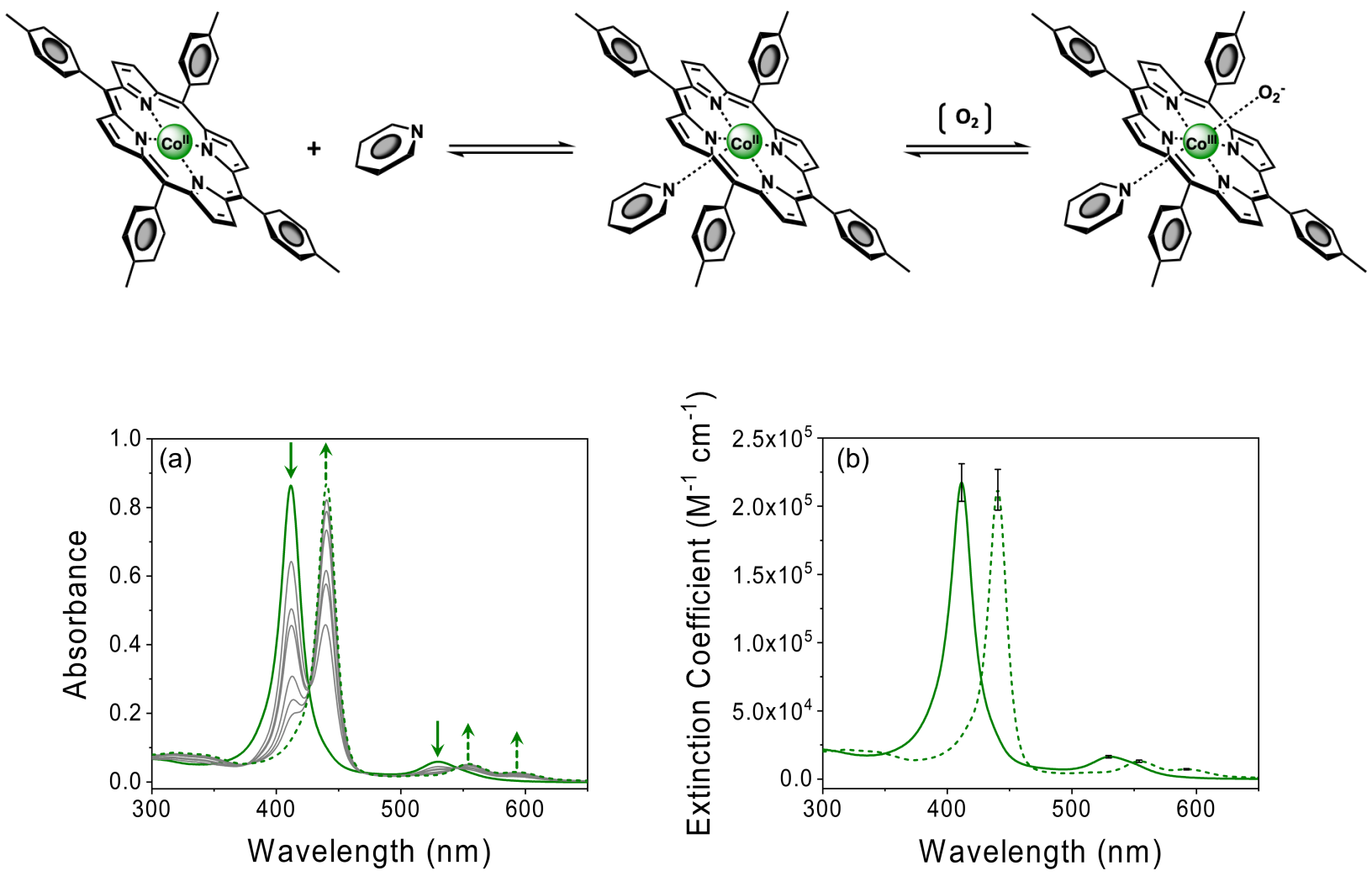

Figure S3. (a) Absorption spectra of 5,10,15,20-tetra- $p$-tolylporphyrin cobalt(II) (CoTTP) in chloroform recorded prior to (solid green) and following 26 hours after the addition of $\sim 1$ equivalent of pyridine (dashed green). The gray lines show data recorded following $5 \mathrm{~min}, 1 \mathrm{~h}, 2 \mathrm{~h}, 7 \mathrm{~h}, 10 \mathrm{~h}$, and $12 \mathrm{~h}$ after the addition of $\sim 1$ equivalent of pyridine. (b) Extinction coefficients of CoTTP in chloroform recorded prior to (solid green) and following 26 hours after the addition of $\sim 1$ equivalent of pyridine (dashed green).

Table S4. Soret and Q-type absorption maxima $\left(\lambda_{\mathrm{abs}}\right)$ as well as associated extinction coefficients $(\varepsilon)$ of 5,10,15,20-tetra- $p$ tolylporphyrin cobalt(II) (CoTTP) in chloroform recorded prior to and following 26 hours after the addition of $\sim 1$ equivalent of pyridine.

\begin{tabular}{c|c|c}
\hline Complex & $\begin{array}{c}\text { Soret Absorption } \\
\lambda_{\text {abs }}(\mathbf{n m}) ; \boldsymbol{\varepsilon}\left(\mathbf{x} \mathbf{1 0}^{\mathbf{5}} \mathbf{M}^{-1} \mathbf{c m}^{-\mathbf{1}}\right)\end{array}$ & $\begin{array}{c}\text { Q-type Absorption(s) } \\
\lambda_{\text {abs }}(\mathbf{n m}) ; \boldsymbol{\varepsilon}\left(\mathbf{x} \mathbf{1 0}^{\mathbf{4}} \mathbf{M}^{-1} \mathbf{c m}^{-1}\right)\end{array}$ \\
\hline CoTTP & $412 ; 2.2 \pm 0.1$ & $530 ; 1.6 \pm 0.1$ \\
CoTTP with $\sim \mathbf{1}$ equivalent of pyridine & $441 ; 2.1 \pm 0.2$ & $554 ; 1.3 \pm 0.1$ and $592 ; 0.72 \pm 0.05$
\end{tabular}



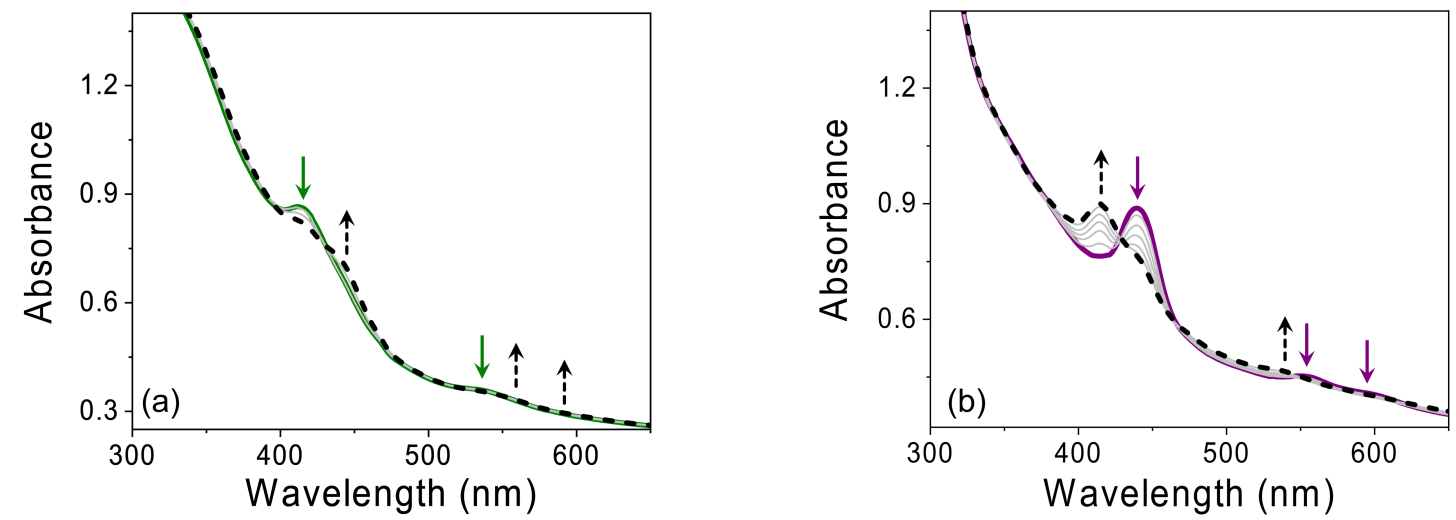

Figure S4. (a) Absorption spectra of cobalt porphyrin-modified nanostructured indium tin oxide (CoP|nanoITO) at open circuit potential (green solid) and polarized at potentials required to generate the $\mathrm{Co}^{\mathrm{III}}$ species (grey solid and black dashed). (b) Absorption spectra of cobalt porphyrin-polyvinylpyridine-modified nanostructured indium tin oxide (CoTTP|PVP|nanoITO) at open circuit potential (purple solid) and polarized at potentials required to generate the $\mathrm{Co}^{\mathrm{II}}$ species (grey solid and black dashed). The black dashed traces in both panels represent spectra of CoP|nanoITO and CoTTP|PVP|nanoITO electrodes that no longer change upon further biasing the applied potential. This data is taken from reference 13 of this supporting information document.

Table S5. Soret and Q-type absorption maxima $\left(\lambda_{\text {abs }}\right)$ as well as associated extinction coefficients $(\varepsilon)$ recorded using $\mathrm{CoP} \mid$ nanoITO or CoTTP|PVP|nanoITO electrodes in propylene carbonate as described in reference 13 of this supporting information document.

\begin{tabular}{c|c|c}
\hline $\begin{array}{c}\text { Heterogeneous-Homogeneous } \\
\text { Construct }\end{array}$ & $\begin{array}{c}\text { Soret Absorption } \\
\lambda_{\text {abs }}(\mathbf{n m}) ; \boldsymbol{\varepsilon}\left(\mathbf{x} \mathbf{1 0}^{4} \mathbf{M}^{-1} \mathbf{c m}^{-1}\right)\end{array}$ & $\begin{array}{c}\text { Q-type Absorption(s) } \\
\lambda_{\text {abs }}(\mathbf{n m}) ; \boldsymbol{\varepsilon}\left(\mathbf{x} \mathbf{1 0}^{4} \mathbf{M}^{-1} \mathbf{c m}^{-1}\right)\end{array}$ \\
\hline CoP|nanoITO & $414 ; 6.1 \pm 0.3$ & $533 ; 1.5 \pm 0.1$ \\
CoTTP|PVP|nanoITO & $439 ; 2.8 \pm 0.6$ & $555 ; 0.45 \pm 0.09$ and $592 ; 0.36 \pm 0.07$
\end{tabular}

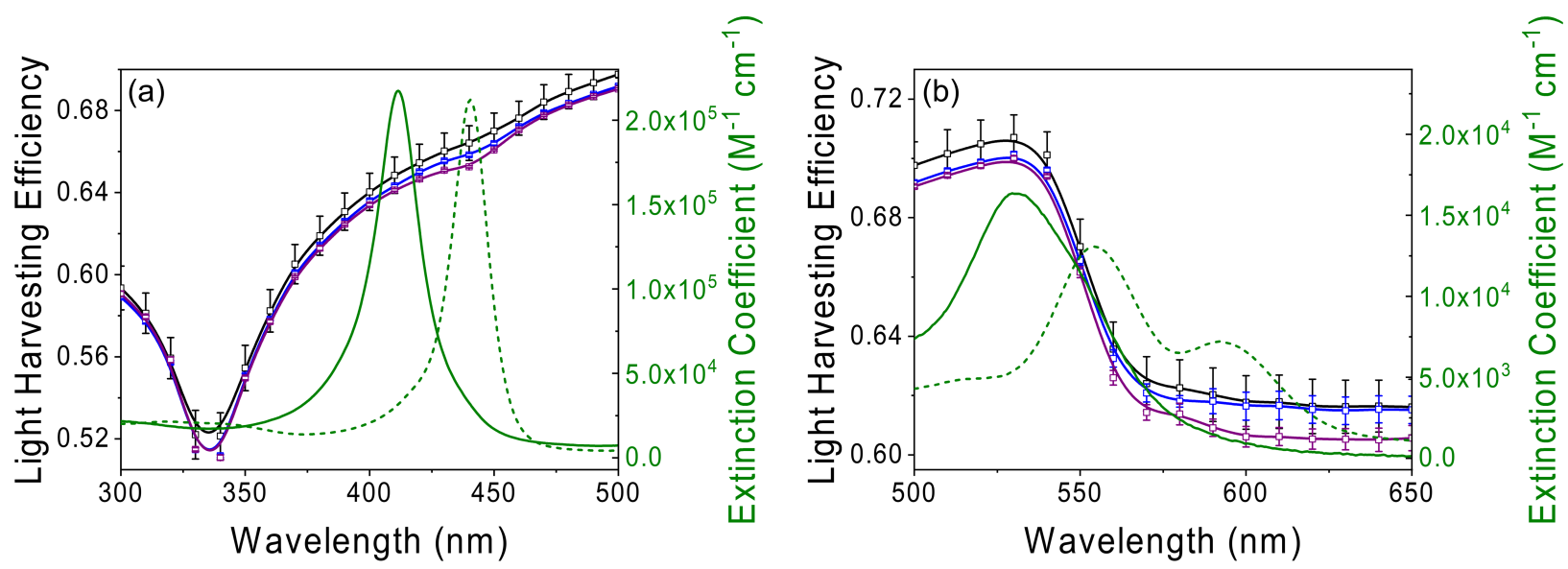

Figure S5. Plots of the light harvesting efficiency measured using GaP (black), PPy $\mid \mathrm{GaP}$ (blue), or CoTTP $|\mathrm{PPy}| \mathrm{GaP}$ (purple) wafers and extinction coefficients of CoTTP in chloroform recorded in the absence (solid green) or presence of $\sim 1$ equivalent of pyridine (dashed green) versus the wavelength of illumination. The data were collected at wavelengths where (a) porphyrin Soret absorption occurs (300-500 nm) and (b) porphyrin Q-type absorptions occur (500-650 nm). 


\section{Approximation of the LHE Associated with the Cobalt Porphyrin-Polypyridyl Layer}

The LHE associated with the cobalt porphyrin-polypyridyl layer (CoTTP|PPy) was approximated using information on the optical properties of CoTTP in chloroform recorded in the presence of $\sim 1$ equivalent of pyridine ${ }^{14}$ as expressed in Equation S9:

$$
\text { LHE of CoTTP|PPy } \approx 1-10^{-\varepsilon l c}
$$

where $\varepsilon$ is the wavelength-dependent extinction coefficient (in units of $\mathrm{M}^{-1} \mathrm{~cm}^{-1}$ ) of CoTTP in chloroform recorded in the presence of $\sim 1$ equivalent of pyridine, $l$ is the thickness (in units of $\mathrm{cm}$ ) of the polypyridyl layer measured on the surfaces of CoTTP $|\mathrm{PPy}| \mathrm{GaP}$, and $c$ is the concentration (in units of $\mathrm{M}$ ) of cobalt porphyrins immobilized on the surfaces of CoTTP $|\mathrm{PPy}| \mathrm{GaP}$.
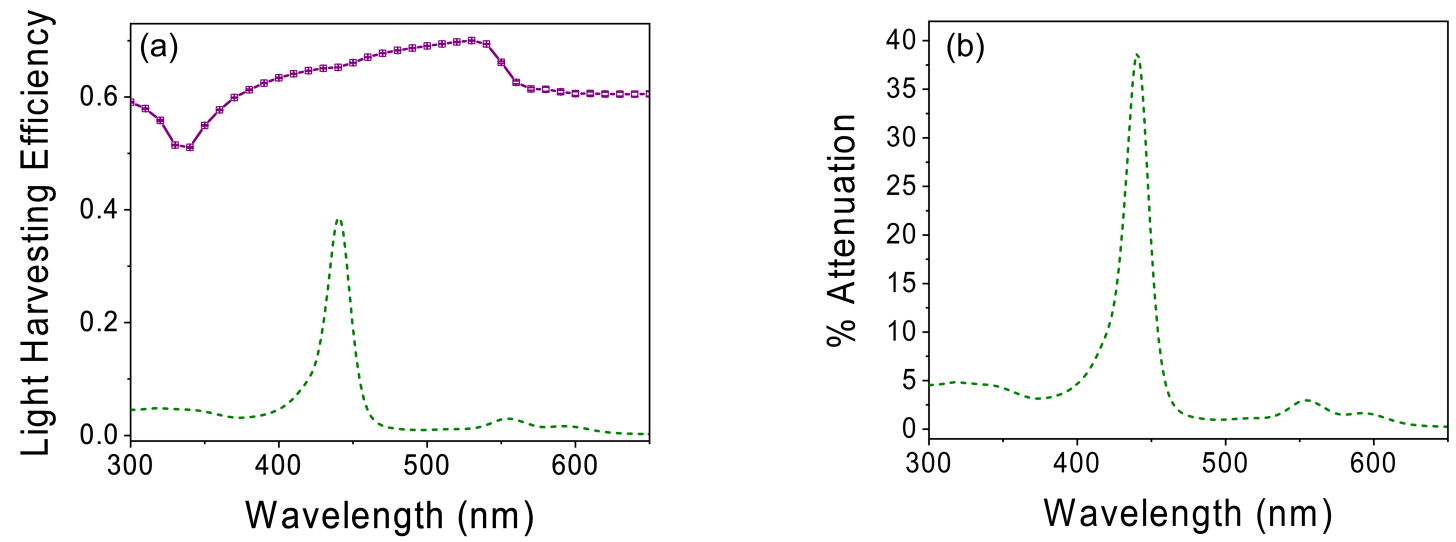

Figure S6. (a) Plots of the light harvesting efficiency (LHE) measured using CoTTP $|\mathrm{PPy}| \mathrm{GaP}$ electrodes (purple solid) and an approximated LHE associated with the CoTTP|PPy layer (green dashed) versus the wavelength of illumination. As expressed in Equation S9, the LHE associated with the CoTTP|PPy layer is approximated using the measured per geometric area loading of cobalt porphyrins on CoTTP $|\mathrm{PPy}| \mathrm{GaP}$ surfaces $\left(1 \mathrm{nmol} \mathrm{cm}{ }^{-2}\right)$, the polypyridyl layer thickness on CoTTP $|\mathrm{PPy}| \mathrm{GaP}$ surfaces $(\sim 4 \mathrm{~nm})$, and the extinction coefficients of CoTTP in chloroform recorded in the presence of $\sim 1$ equivalent of pyridine. (b) Plot of the percent of light attenuated by the cobalt porphyrin-polypyridyl coating of the CoTTP $|\mathrm{PPy}| \mathrm{GaP}$ assemblies versus the wavelength of illumination. Using the LHE values associated with the CoTTP $\mid$ PPy layer from panel a, the percent of light attenuated by the cobalt porphyrin-polypyridyl coating is estimated to be $\sim 39 \%$ at the wavelength associated with the Soret absorption maximum at $441 \mathrm{~nm}, \sim 3 \%$ at the wavelength associated with the Qtype absorption maximum at $554 \mathrm{~nm}$, and $\sim 2 \%$ at the wavelength associated with the Q-type absorption maximum at 592 nm. 


\section{External Quantum Efficiency Action Spectra}

Hypothetical EQE action spectra associated with CoTTP $|\mathrm{PPy}| \mathrm{GaP}$ electrodes (red and teal spectra in Figures S7 and S8) were constructed assuming the immobilized porphyrins serve strictly as pigments or dye sensitizers, and not as catalysts.

\section{Hypothetical External Quantum Efficiency Action Spectra Assuming 0\% Dye-Contribution Efficiency}

The hypothetical EQE of CoTTP $|\mathrm{PPy}| \mathrm{GaP}$ if the dye-contribution efficiency (DCE) is 0\% (Hypothetical EQE of CoTTP $|\mathrm{PPy}| \mathrm{GaP}_{0 \% \mathrm{DCE}}$ ) was constructed by multiplying the EQE measured using samples of PPy|GaP (blue action spectrum in Figure 4a) by one minus the LHE associated with the CoTTP|PPy layer (dashed green spectrum in Figure S6a) as expressed in Equation S10:

Hypothetical EQE of CoTTP $|\mathrm{PPy}| \mathrm{GaP}_{0 \% \mathrm{DCE}} \approx$ Measured EQE of PPy $\mid \mathrm{GaP} \bullet(1-\mathrm{LHE}$ of CoTTP $\mid \mathrm{PPy})$

When the dye-contribution efficiency is $0 \%$, every photon absorbed by the CoTTP|PPy layer is screened from the underlying GaP and thus do not contribute to production of current.

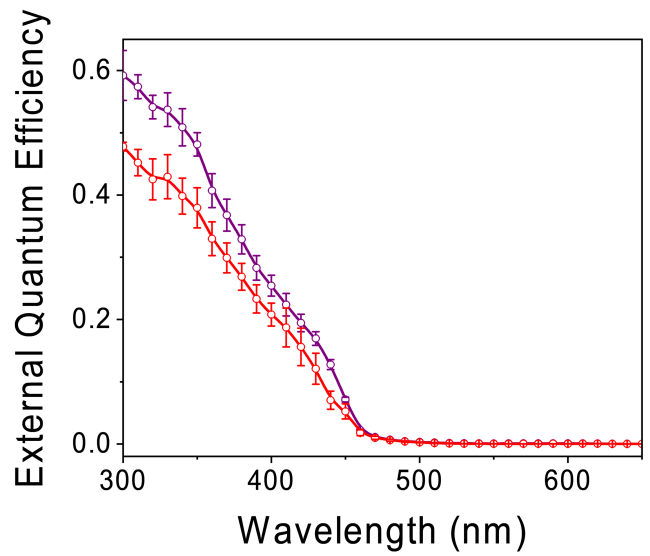

Figure S7. External quantum efficiency (EQE) action spectrum recorded using CoTTP $|\mathrm{PPy}| \mathrm{GaP}$ electrodes polarized at $0 \mathrm{~V}$ vs RHE in $0.1 \mathrm{M}$ phosphate buffer ( $\mathrm{pH} 7$ ) (purple) and a hypothetical EQE action spectrum of CoTTP|PPy|GaP electrodes assuming the immobilized porphyrins serve strictly as pigments, not catalysts, and that all photons absorbed by the porphyrin layer are screened from the underlying $\mathrm{GaP}$ and do not contribute to production of current (i.e., the DCE is $0 \%$ ) (red). 


\section{Hypothetical External Quantum Efficiency Action Spectra Assuming 100\% Dye-Contribution Efficiency}

The hypothetical EQE of the CoTTP|PPy layer (Hypothetical EQE of CoTTP|PPy) is defined as the product of the LHE of CoTTP|PPy (dashed green spectrum in Figure S6a) and the dye-contribution efficiency (DCE) as defined according to Equation S11:

Hypothetical EQE of CoTTP $\mid \mathrm{PPy}=\mathrm{LHE}$ of CoTTP $\mid \mathrm{PPy} \bullet \mathrm{DCE}$

The dye-contribution efficiency in Eq S11 is unity when the dye-contribution efficiency associated with the CoTTP|PPy layer is $100 \%$. Thus, the hypothetical EQE of CoTTP|PPy shown in Equation S12 (Hypothetical EQE of CoTTP|PPy $100 \%$ DCE) is equal to the LHE of CoTTP|PPy:

Hypothetical EQE of CoTTP $\mid \mathrm{PPy} 100 \%$ DCE $=$ LHE of CoTTP $\mid \mathrm{PPy} \bullet 1$

The hypothetical EQE of CoTTP $|\mathrm{PPy}| \mathrm{GaP}$ if the dye-contribution efficiency is 100\% (Hypothetical EQE of CoTTP $\left.|\mathrm{PPy}| \mathrm{GaP}_{100 \% \text { DCE}}\right)$ was constructed by summing the hypothetical EQE of CoTTP $|\mathrm{PPy}| \mathrm{GaP}_{0 \%}$ DCE and the hypothetical EQE of CoTTP $\mid$ PPy $100 \%$ DCE as expressed in Equation S13:

Hypothetical EQE of CoTTP $|\mathrm{PPy}| \mathrm{GaP}_{100 \% \text { DCE }} \approx$ Hypothetical EQE of CoTTP $|\mathrm{PPy}| \mathrm{GaP}_{0 \%} \mathrm{DCE}+$ Hypothetical EQE of CoTTP $\mid \mathrm{PPy}_{100 \% \text { DCE }}$

When the dye-contribution efficiency is $100 \%$, every photon absorbed by the CoTTP|PPy layer is screened from the underlying GaP but contributes to production of current via a dye-sensitization mechanism.

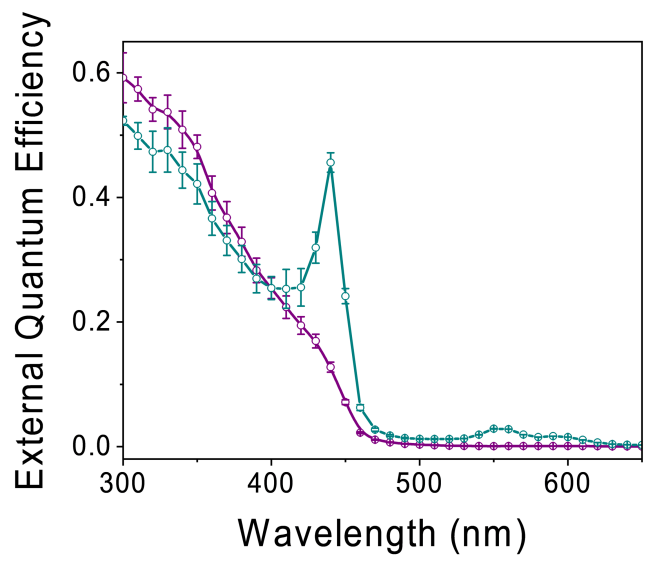

Figure S8. External quantum efficiency (EQE) action spectrum recorded using CoTTP $|\mathrm{PPy}| \mathrm{GaP}$ electrodes polarized at $0 \mathrm{~V}$ vs RHE in $0.1 \mathrm{M}$ phosphate buffer ( $\mathrm{pH} 7$ ) (purple) and a hypothetical EQE action spectrum of CoTTP|PPy|GaP electrodes assuming the immobilized porphyrins serve strictly as pigments, not catalysts, and that all photons absorbed by the porphyrin layer are screened from the underlying $\mathrm{GaP}$ but contribute to production of current via a dye-sensitization mechanism (i.e., the DCE is $100 \%$ ) (teal). 


\section{Apparent Dye-Contribution Efficiencies}

The EQEs measured using samples of CoTTP $|\mathrm{PPy}| \mathrm{GaP}$ can be expressed as a sum of the hypothetical EQEs associated with photons striking the cobalt porphyrin-polypyridyl coating or the underlying semiconductor in accordance with Equation S14:

Measured EQE of CoTTP $|\mathrm{PPy}| \mathrm{GaP} \approx$ Hypothetical EQE of CoTTP $|\mathrm{PPy}| \mathrm{GaP}_{0 \% \mathrm{DCE}}+$ Hypothetical EQE of CoTTP $\mid \mathrm{PPy}$

If the measured enhancements in EQE following porphyrin modification are solely due to dye sensitization, Equation S14 can be re-expressed (using the relationship given in Equation S11) in terms of an apparent DCE required to afford the measured EQE values, as indicated in Equation S15:

Measured EQE of CoTTP $|\mathrm{PPy}| \mathrm{GaP} \approx$ Hypothetical EQE of CoTTP $|\mathrm{PPy}| \mathrm{GaP}_{0 \% \mathrm{DCE}}+(\mathrm{LHE}$ of CoTTP $\mid \mathrm{PPy} \bullet \mathrm{Apparent} \mathrm{DCE})$

This apparent DCE is approximated by rearranging Equation S15 to yield Equation S16:

Apparent DCE $\approx \frac{\text { Measured EQE of CoTTP }|\mathrm{PPy}| \mathrm{GaP}-\text { Hypothetical EQE of CoTTP }|\mathrm{PPy}| \mathrm{GaP}_{0 \% \text { DCE }}}{\text { LHE of CoTTP } \mid \mathrm{PPy}}$

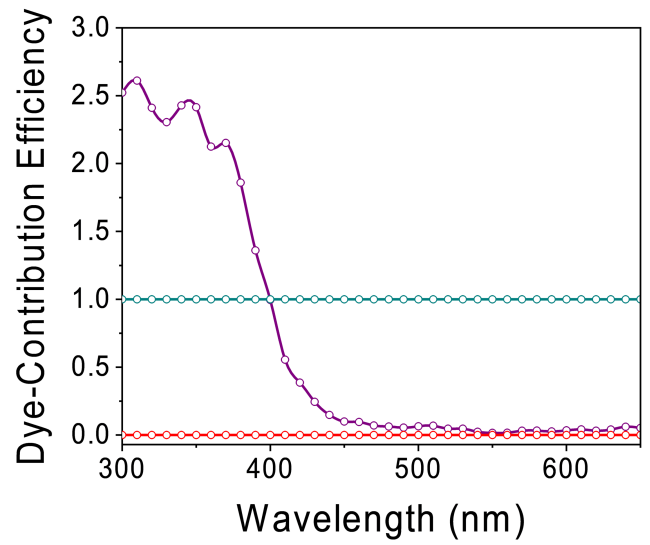

Figure S9. A plot of the apparent dye-contribution efficiency (DCE) (as defined in Equation S16) (purple) required to achieve the external quantum efficiency values (EQE) measured using CoTTP $|\mathrm{PPy}| \mathrm{GaP}$ electrodes versus the wavelength of illumination. For comparisons, the DCEs used to construct the hypothetical EQE action spectra shown in Figure S7, where the DCE is $0 \%$, (red) and Figure S8, where the DCE is $100 \%$ (teal) are also included. These results further demonstrate that enhancements in EQE following porphyrin modification cannot be accounted for by porphyrin dye sensitization. For example, the apparent DCEs required to achieve the EQEs measured following porphyrin modification exceed $250 \%$. 


\section{Spectral Profile of the LSC-100 Series Oriel Solar Simulator}
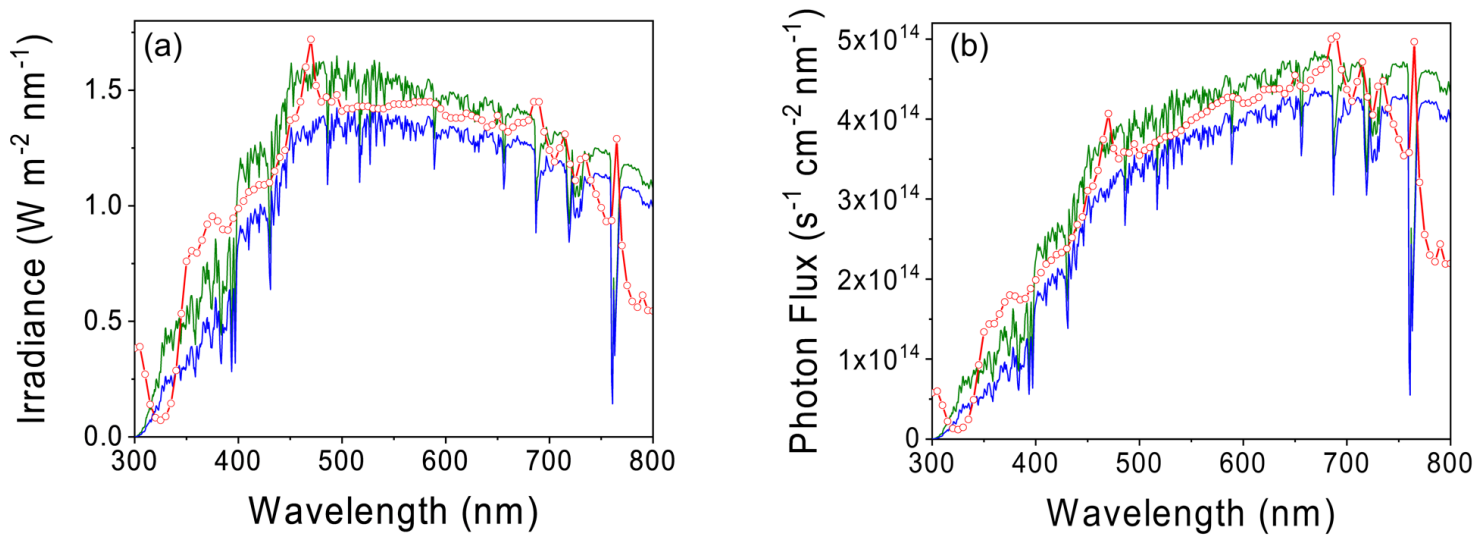

Figure S10. (a) Irradiance and (b) photon flux spectra of an LSC-100 Series Oriel Solar Simulator collected with an air mass 1.5 filter (red circles). For comparison, the air mass 1.5 global tilt (green) and direct circumsolar (blue) irradiance and photon flux spectra are included. ${ }^{12}$ 


\section{Supporting Information References}

(1) Khusnutdinova, D.; Beiler, A. M.; Wadsworth, B. L.; Jacob, S. I.; Moore, G. F. Metalloporphyrin-Modified Semiconductors for Solar Fuel Production. Chem. Sci. 2017, 8, 253-259.

(2) Lee, C. H.; Lindsey, J. S. One-Flask Synthesis of Meso-Substituted Dipyrromethanes and Their Application in the Synthesis of Trans-Substituted Porphyrin Building Blocks. Tetrahedron 1994, 50, 11427-11440.

(3) Chizhova, N. V.; Kumeev, R. S.; Mamardashvili, N. Z. Synthesis and Spectral Properties of Cobalt (II) and Cobalt (III) Tetraarylporphyrinates. Russ. J. Inorg. Chem. 2013, 58, 740-743.

(4) Beiler, A. M.; Khusnutdinova, D.; Wadsworth, B. L.; Moore, G. F. Cobalt Porphyrin-Polypyridyl Surface Coatings for Photoelectrosynthetic Hydrogen Production. Inorg. Chem. 2017, 56, 12178-12185.

(5) Krawicz, A.; Yang, J.; Anzenberg, E.; Yano, J.; Sharp, I. D.; Moore, G. F. Photofunctional Construct that Interfaces Molecular Cobalt-Based Catalysts for $\mathrm{H}_{2}$ Production to a Visible-Light-Absorbing Semiconductor. J. Am. Chem. Soc. 2013, 135, 11861-11868.

(6) Cedeno, D.; Krawicz, A.; Doak, P.; Yu, M.; Neaton, J. B.; Moore, G. F. Using Molecular Design to Control the Performance of Hydrogen-Producing Polymer-Brush-Modified Photocathodes. J. Phys. Chem. Lett. 2014, 5, 32223226.

(7) Beiler, A. M.; Khusnutdinova, D.; Jacob, S. I.; Moore, G. F. Solar Hydrogen Production Using Molecular Catalysts Immobilized on Gallium Phosphide (111)A and (111)B Polymer-Modified Photocathodes. ACS Appl. Mater. Interfaces 2016, 8, 10038-10047.

(8) Beiler, A. M.; Khusnutdinova, D.; Jacob, S. I.; Moore, G. F. Chemistry at the Interface: Polymer-Functionalized GaP Semiconductors for Solar Hydrogen Production. Ind. Eng. Chem. Res. 2016, 55, 5306-5314.

(9) Walker, F. A. Electron Spin Resonance Study of Coordination to the Fifth and Sixth Positions of $\alpha, \beta, \gamma, \delta$-Tetra(pmethoxyphenyl)porphinatocobalt (II). J. Am. Chem. Soc. 1970, 92, 4235-4244.

(10) Walker, F. A. Reactions of Monomeric Cobalt-Oxygen Complexes. I. Thermodynamics of Reaction of Molecular Oxygen with Five-and Six-Coordinate Amine Complexes of a Cobalt Porphyrin. J. Am. Chem. Soc. 1973, 95, 11541159.

(11) Honda, S.; Ohkita, H.; Benten, H.; Ito, S. Multi-Colored Dye Sensitization of Polymer/Fullerene Bulk Heterojunction Solar Cells. Chem. Commun. 2010, 46, 6596-6598.

(12) At the time of writing, the air mass 1.5 global tilt (AM 1.5 G) spectrum from the ASTM G-173-03 data set is available online at https://www.nrel.gov/grid/solar-resource/spectra.html (accessed November 12, 2019).

(13) Wadsworth, B. L.; Khusnutdinova, D.; Urbine, J. M.; Reyes, A. S.; Moore, G. F. Expanding the Redox Range of Surface-Immobilized Metallocomplexes using Molecular Interfaces. ACS Appl. Mater. Interfaces 2020, 12, 39033911

(14) This analysis does not account for changes in the optical properties of the underlying GaP or cobalt porphyrin species following their surface immobilization. 